\title{
RELAÇÕES ENTRE APTIDÃO FÍSICA, CONCENTRAÇÃO DE CREATINA, QUINASE E VARIABILIDADE DA FREQUÊNCIA CARDÍACA EM ALUNOS DO NPOR DE PELOTAS/RS 1
}

\author{
Fabrício Boscolo Del Vecchio \\ Universidade Federal de Pelotas, Pelotas, Rio Grande do Sul, Brasil \\ Luis Felipe Oliveira \\ Universidade Federal de Pelotas, Pelotas, Rio Grande do Sul, Brasil \\ Hector Ferreira \\ Universidade Federal de Pelotas, Pelotas, Rio Grande do Sul, Brasil \\ Jonathan Barth \\ Universidade Federal de Pelotas, Pelotas, Rio Grande do Sul, Brasil \\ Victor Silveira Coswig \\ Universidade Federal de Pelotas, Pelotas, Rio Grande do Sul, Brasil
}

\begin{abstract}
Resumo
Objetivou-se avaliar o perfil de militares da ativa do Núcleo de Preparação dos Oficiais da Reserva de Pelotas/RS. Foram envolvidos 30 militares que cumpriam treinamento físico diário. Mensurou-se potência de membros inferiores, velocidade, resistência de força, potência aeróbia (VO2max), variabilidade da frequência cardíaca (VFC) e concentração de creatina quinase ([CK]). Empregouse a estatística descritiva e as correlações foram avaliadas com teste de Pearson. Os envolvidos

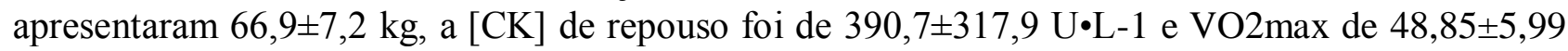
$\mathrm{mL} \cdot \mathrm{kg}-1 \cdot \mathrm{min}-1$. Conclui-se que a aptidão física dos militares envolvidos é intermediária. A concentração de CK está próxima ao limite superior de referência e os valores da VFC indicam funcionamento autonômico adequado.
\end{abstract}

Palavras-chave: Aptidão física, Militares, Educação física e treinamento, Creatina quinase, Frequência cardíaca

\section{Introdução}

No Brasil, são raros os estudos com avaliação da aptidão física de militares ingressantes (LEVANDOSKI et al., 2013) ou de carreira nas forças armadas (JÚNIOR; KRUG, 2011). Por outro lado, aponta-se a necessidade de bom condicionamento físico e rápida capacidade de mobilização por parte destes indivíduos, com vistas a manejar os diferentes distúrbios sociais, inclusive guerras (LINDQUIST; BRAY, 2001). Isto se dá, pois conflitos no mundo aumentam em número e em 
intensidade (HENDERSON et al., 2000), e o Brasil tem: i) recrutado militares para controle de transtornos urbanos (ERLACHER, 2009), ii) contribuído com pacificação em outros países, como o Haiti (SEITENFUS, 2006) e iii) cogitado inserção permanente no Conselho de Segurança das Nações Unidas (RESENDE; GONÇALVES, 2009).

Níveis elevados de aptidão física entre militares são relevantes para a segurança pública e da nação e, em âmbito nacional, no primeiro ano de ingresso, recrutas passam por treinamento intenso para aprimorar suas capacidades físicas e possuírem excelência militar (ROBERT et al., 2011). Porém, internacionalmente, observa-se que a aptidão física dos soldados, após doze meses de serviço militar, diminui significativamente quando comparada à do ano de conscrito (DREGVAL; VAICAITIENÉ, 2006). De modo complementar, indica-se que, durante o treinamento básico, primeiros 2-3 meses de serviço militar, a potência aeróbia máxima $\left(\mathrm{VO}_{2 \mathrm{max}}\right)$ de recrutas que se apresentaram com valores iniciais baixos tende a aumentar; no entanto, depois do treinamento básico, o $\mathrm{VO}_{2} \max$ de todos os militares passa a diminuir, independente dos valores iniciais (DYRSTAD; SOLTVEDT; HALLE'N, 2006).

Complementarmente, ao se realizarem testes com elevadas exigências, é possível identificar militares com melhor aptidão física. Aponta-se ainda que a variabilidade da frequência cardíaca (VFC), que é considerada como indicador prévio de riscos à saúde e utilizada para avaliar as adaptações ao exercício (OLIVEIRA et al., 2012), pode ser empregada na avaliação do funcionamento adequado de mecanismos de controle autonômico, e valores diminutos da mesma tendem a representar comprometimento de funções fisiológicas (PUMPRLA et al., 2002; CARTER et al., 2003). A concentração de creatina-quinase ([CK]) tem sido empregada como indicador de dano muscular proveniente do alto grau de intensidade de esforços físicos e, possivelmente, de lesões causadas por impactos corporais no treinamento (BARTONE et al., 2008). Inclusive, a partir da $[\mathrm{CK}]$ que se diagnostica rabdomiólise, síndrome que envolve falha renal e pode conduzir a óbito, e que exibiu aumento de 30\% no número de casos entre militares norteamericanos entre 2008-2012 (MSMR, 2012). Ademais, constata-se que para a formação de militares de elite é necessário desenvolvimento adequado de componentes físicos e fisiológicos (THOMAS et al., 2004; HARTMANN et al., 2003), embora a relação entre eles ainda seja pouco estudada. Assim, o objetivo do presente estudo foi avaliar o nível de aptidão física e o estado fisiológico de militares da ativa, após período básico de treinamento e anterior à realização da primeira missão de campo operacional.

\section{Materiais e Métodos}

Tipo de estudo e amostra

O estudo se caracteriza como observacional descritivo. Quanto aos aspectos éticos, os militares assinaram termo de consentimento livre e esclarecido (TCLE), o estudo foi autorizado pelo pavilhão de comando do batalhão e conta com aprovação no comitê de ética local (CEP protocolo \#004/2012).

Para a realização deste estudo, foram envolvidos 30 militares da ativa, alunos do Núcleo de Preparação de Oficiais da Reserva/NPOR, todos do sexo masculino e maiores de 18 anos. Os sujeitos eram ingressantes no serviço militar no corrente ano da pesquisa e no período de realização do estudo já haviam completado três meses de treinamento, que, segundo o quadro de trabalho 
semanal do batalhão, constituía-se de uma hora diária ou mais de treinamento físico militar (TFM). O cálculo do tamanho amostral se deu considerando estudo prévio que encontrou coeficiente de correlação de 0,53 entre potência aeróbia máxima e medida de índice vagal do domínio da frequência da VFC = HF/(LF+HF) (BUCHHEIT; GINDRE, 2006). Assim, considerando-se este valor de r, poder de $80 \%$ e nível de significância de 5\%, seriam necessários 27 sujeitos. Optou-se pela inclusão de $10 \%$ dada a chance de perda amostral durante os procedimentos, a qual não se concretizou.

\section{Delineamento do estudo}

Para realização das atividades, os envolvidos foram: i) convidados a assistir palestra explicativa prévia (com sete dias de antecedência), ii) comunicados dos procedimentos com $24 \mathrm{~h}$ de antecedência, iii) alertados para não realizarem atividades físicas moderadas ou vigorosas nas 24 horas antecedentes às avaliações e não alterarem seus padrões alimentares e de sono-vigília, iv) chamados a realizarem todos procedimentos, que ocorreram na parte da manhã, entre $10 \mathrm{~h}$ e $12 \mathrm{~h}$. Com vistas ao envolvimento do maior número possível de pessoas, a investigação foi delineada para ocorrer em dia único, e toda estrutura laboratorial e avaliativa foi instalada na unidade do batalhão.

Após a autorização do comandante do batalhão e a assinatura do TCLE, os alunos foram submetidos a uma bateria de avaliações fisiológicas e antropométricas, testes físicos, conduzidos por uma equipe previamente treinada, com um único avaliador e anotador sendo responsável por cada procedimento avaliativo.

\section{Procedimentos de coleta e registro dos dados}

Os dados foram anotados em formulários individuais e, após, transferidos para planilhas eletrônicas. Durante a coleta dos dados, foram realizados os seguintes procedimentos:

Medidas antropométricas: Foram medidas massa corporal, estatura e se tomaram os valores de três dobras cutâneas (Peitoral, Abdômen e Coxas), mensuradas três vezes e em sistema de rodízio, com emprego de adipômetro científico (CESCORF®, São Paulo, Brasil). Considerou-se o valor de densidade corporal, obtido a partir do protocolo de três dobras cutâneas (JACKSON \& POLLOCK, 1978), para que fosse estimado o percentual de gordura usando a equação de Siri (1961).

Concentração de Creatina Quinase [CK]: Logo após as medidas antropométricas e antes dos testes motores, os sujeitos do estudo foram submetidos a coleta sanguínea para mensuração da [CK]. Todos os procedimentos foram executados por profissional com mais de três anos de experiência, empregando-se luvas de procedimento e lancetas descartáveis. Para a [CK] sanguínea, extraíram-se $32 \mu \mathrm{L}$ de sangue capilar, a partir de punção no lóbulo da orelha, após higienização prévia com álcool etílico a 95\%. A amostra de sangue foi coletada por tubo capilar e pipetada em fita reagente (Registro ANVISA 10287410157, Reflotron $\left.{ }^{\circledR}\right)$, a qual foi inserida e lida por analisador portátil, a partir de fotometria de reflectância (Reflotron Analyser ${ }^{\circledR}$, Boehringer-Mannheim, França).

Força isométrica de preensão manual (FIPM): Para avaliação da FIPM, os sujeitos adotaram posição ortostática e, com cotovelos estendidos e membros superiores ao longo do corpo, pressionaram dinamômetro JAMAR ${ }^{\mathrm{TM}}$ (Sammons Preston, EUA) o mais forte quanto possível por 5 
segundos. Foram realizadas três medições para cada sujeito com intervalo de um minuto entre cada uma e a média dessas medidas foi considerada na análise dos dados. Este procedimento exibe coeficiente de correlação intraclasse de 0,93 e alta reprodutibilidade (ZANCHET; DEL VECCHIO, 2013).

Potência muscular de membros inferiores: Conduziram-se dois testes, um de salto vertical (SV) com contramovimento (reprodutibilidade teste-reteste com $r=0,93$; MARKOVIC et al., 2004), com tapete específico (Jump System, CEFISE®, Nova Odessa), e de salto horizontal, com a utilização de trena metálica inelástica de $5 \mathrm{~m}$ ( $\mathrm{r}=0,83$ a 0,99; CASTRO-PIÑERO et al., 2010). Foram executadas três tentativas para cada teste e a média foi considerada nas análises.

Velocidade de corrida: Foi realizado teste de velocidade de $20 \mathrm{~m}$, utilizando fotocélulas (Kit MultiSprint Full, Hidrofit ${ }^{\circledR}$, Belo Horizonte). Foram posicionados três pontos de registro, na largada, a $10 \mathrm{~m}$ e $20 \mathrm{~m}$, sendo que os alunos foram instruídos a desacelerarem apenas após cruzarem a última fotocélula (PIMENTA, 2012).

Resistência de força: Foram realizados quatro testes (HARMAN et al., 2008): 1) Agachamento, os sujeitos foram submetidos a dois minutos de agachamentos ininterruptos, com dedos entrelaçados na altura da nuca e solicitados flexionarem os joelhos em angulação inferior a $90^{\circ}$; 2) Flexão de braços, em 2 minutos, os alunos deveriam realizar o maior número de repetições possíveis, partindo de decúbito ventral, com ombros abduzidos e com os cotovelos estendidos, apenas com palmas das mãos e as pontas dos pés em apoio ao solo, realizando flexão dos cotovelos até, aproximadamente, $90^{\circ}$ e, então, retornando a posição inicial; 3) Abdominais, em decúbito dorsal, joelhos flexionados e plantas dos pés apoiadas no solo, sem suporte, com as mãos na nuca, deveriam elevar o tronco até aproximar os cotovelos do joelho e retornar a posição inicial o maior número de vezes em 2 min; 4) Barra Fixa, registrou-se a quantidade máxima de repetições que os alunos executaram de maneira ininterrupta, as únicas exigências foram: mãos deveriam estar em posição pronada e o queixo necessitaria passar da altura da barra para ser considerada repetição válida.

Potência aeróbia máxima $\left(\mathrm{VO}_{2 \max }\right)$ : Foi realizada corrida de $3200 \mathrm{~m}$, na qual os avaliados deveriam completar esta distância no menor tempo possível. $\mathrm{O} \mathrm{VO}_{2 \max }$ foi calculado segundo a equação: $\mathrm{VO}_{2 \max }\left(\mathrm{mL} \cdot \mathrm{kg}^{-1} \cdot \mathrm{min}^{-1}\right)=118,4-4,774 \cdot(\mathrm{T})$, sendo $\mathrm{T}=$ tempo em minutos e com fração decimal (WELTMAN, 1989).

Flexibilidade: Para análise da flexibilidade, empregou-se o teste de sentar e alcançar de Wells, no qual os sujeitos deveriam sentar no chão com as plantas dos pés unidas e apoiadas no banco, mão direita sobre a esquerda e, com cotovelos estendidos, tentaram alcançar o mais longe possível, sem flexionar os joelhos (WELLS; DILLON, 1952).

Frequência cardíaca (FC) e Variabilidade da frequência cardíaca (VFC): Para registro da VFC, empregou-se monitor cardíaco (Polar ${ }^{\circledR}$, modelo RS800) e cinta torácica (BARTH; DEL VECCHIO, 2014). Para coleta e registro dos dados, o sujeito permaneceu sentado e imóvel por 5 min (VANDERLEI et al., 2009). Foram consideradas variáveis dos domínios: a) de Frequência, alta, baixa, muito baixa, respectivamente HF, LF e VLF, assim como relação LF/HF; b) do Tempo, com o tempo médio entre dois batimentos cardíacos (MédRR), desvio padrão normal dos intervalos RR (SDNN), a raiz quadrada da média do quadrado das diferenças entre intervalos RR normais adjacentes (RMSSD) e porcentagem de intervalos RR com diferenciação de duração maior que $50 \mathrm{~ms}$ quando comparados aos intervalos adjacentes (PNN50) e f) Não-linear, com as variáveis desvio padrão entre pontos instantâneos (SD1) e desvio padrão de tendência em longo prazo (SD2) 
(OLIVEIRA et al., 2012). Registraram-se os valores máximos e médios, em batimentos por minuto (bpm), da FC de repouso.

\section{Análise estatística}

Inicialmente, conduziu-se teste de normalidade de Shapiro-Wilk. Após, empregou-se estatística descritiva, considerando-se média, desvio padrão (dp) e coeficiente de variação (CV). Testaram-se correlações entre variáveis com a prova produto-momento de Pearson. Assumiu-se 5\% como nível de significância estatística. Para todos os testes foi utilizado software SPSS 17.0.

\section{Resultados}

Os conscritos envolvidos no presente estudo apresentavam massa corporal de 66,9 $\pm 7,2 \mathrm{~kg}$, somatório de três dobras cutâneas de 27,06 $\pm 8,7 \mathrm{~mm}$ e $12,2 \pm 3,3 \%$ de gordura corporal estimada. Os valores da $[\mathrm{CK}]$ de repouso foram de $390,7 \pm 317,9 \mathrm{U} \cdot \mathrm{L}^{-1}$. Os dados de aptidão física são apresentados na tabela 1. Evidencia-se que, com exceção do teste de flexibilidade (35\%) e de repetições na barra fixa $(29,8 \%)$ e flexões de braço $(26,5 \%)$, foi encontrada baixa $(<10 \%)$ ou moderada $(<20 \%)$ variação da distribuição dos dados.

Tabela 1. Medidas descritivas de centralidade e dispersão das variáveis de aptidão física de militares no Núcleo de Preparação Física de Oficiais da Reserva de Pelotas/RS (n=30).

\begin{tabular}{lccc}
\hline \multicolumn{1}{c}{ Componente neuromuscular } & Média & $\mathrm{dp}$ & $\mathrm{CV}(\%)$ \\
Flexibilidade (cm) & & & \\
Salto Vertical (cm) & 24,45 & 8,72 & 35,6 \\
Salto Horizontal (cm) & 27,78 & 3,71 & 13,3 \\
Tempo de Sprint - 10m (s) & 185,10 & 15,16 & 8,2 \\
Tempo de Sprint - 20m (s) & 1,97 & 0,09 & 4,5 \\
FIPM - mão direita (kgf) & 3,38 & 0,16 & 4,7 \\
FIPM - mão esquerda (kgf) & 42,84 & 8,05 & 18,8 \\
Agachamento (reps) & 41,15 & 7,56 & 18,4 \\
Flexões de braço (reps) & 83,82 & 13,23 & 15,8 \\
Abdominais (reps) & 47,83 & 12,66 & 26,5 \\
Barra fixa (reps) & 49,30 & 10,02 & 20,3 \\
Potência aeróbia máxima (mL·kg ${ }^{-1} \cdot \mathrm{min}^{-1}$ ) & 10,23 & 3,05 & 29,8 \\
dp = desvio padrão; CV = coeficiente de variação. & 48,85 & 5,99 & 12,3 \\
\hline
\end{tabular}

Os dados referentes à frequência cardíaca de repouso máxima e média, bem como sua variabilidade, nos diferentes domínios: tempo, frequência e não linear, são apresentados na tabela 2. Nela, expressa-se que domínios VLF e HF exibiram elevada variação interindividual, porém, valores muito baixos do coeficiente de variação foram observados na FC. 
Tabela 2. Medidas descritivas de centralidade e dispersão da frequência cardíaca e variabilidade da frequência cardíaca de militares no Núcleo de Preparação Física de Oficiais da Reserva de Pelotas/RS ( $\mathrm{n}=30)$.

\begin{tabular}{lccc}
\hline & Média & $\mathrm{dp}$ & $\mathrm{CV}(\%)$ \\
\hline Variáveis cardíacas & & & \\
FC de repouso - média (bpm) & 61,36 & 8,25 & 13,4 \\
FC de repouso - máxima (bpm) & 86,26 & 13,21 & 15,3 \\
Variabilidade da Frequência Cardíaca & & & \\
Domínio do tempo & & & \\
MédRR (ms) & 995,31 & 136,59 & 13,7 \\
SDNN (ms) & 107,51 & 32,41 & 30,1 \\
RMSSD (ms) & 87,99 & 38,28 & 43,5 \\
PNN50 (\%) & 44,87 & 16,76 & 37,3 \\
Domínio da frequência & & & \\
VLF (ms ${ }^{2}$ & 4102,69 & 4596,91 & 112,0 \\
LF (ms $)$ & 3376,43 & 1852,49 & 54,8 \\
HF (ms ${ }^{2}$ ) & 2915,29 & 3011,36 & 103,3 \\
LF/HF (\%) & 1,90 & 1,51 & 79,4 \\
Domínio não-linear & & & \\
SD1 (ms) & 62,32 & 27,09 & 43,5 \\
SD2 (ms) & 137,88 & 39,26 & 28,5 \\
\hline
\end{tabular}

MedRR = Média dos intervalos entre batimentos; SDNN = Desvio padrão dos intervalos entre batimentos; RMSSD = Raiz quadrada das médias sucessivas ao quadrado; PNN50 = Percentual dos intervalos entre batimentos com mais de $50 \mathrm{~ms}$ em relação ao subsequente; VLF = Banda de muito baixa frequência; $\mathrm{LF}=$ Banda de baixa frequência; $\mathrm{HF}=$ Banda de alta frequência; $\mathrm{LF} / \mathrm{HF}=\mathrm{Razão}$ entre LF e HF; SD1 = Desvio padrão entre pontos instantâneos; SD2 = Desvio padrão de tendência em longo prazo.

Acerca das correlações, $[\mathrm{CK}]$ e $\mathrm{VO}_{2}$ max não se correlacionaram com nenhuma das demais variáveis mensuradas. Observa-se correlação entre hemicorpos para FIPM $(r=0,88 ; p<0,001)$ e entre marcas no tempo para se cumprir $10 \mathrm{~m}$ e $20 \mathrm{~m}(\mathrm{r}=0,78 ; \mathrm{p}<0,001)$. O desempenho no teste de flexibilidade se correlacionou apenas com a distância obtida no salto horizontal $(\mathrm{r}=0,37 ; \mathrm{p}=$ 0,04). O número de repetições na barra fixa se correlacionou com o resultado nos testes de flexão de braço $(r=0,78 ; p<0,001)$ e de abdominais $(r=0,38 ; p=0,03)$. Este, por sua vez, relacionou-se com o resultado no teste de repetições máximas no agachamento $(r=0,41 ; p=0,02)$.

Quanto às variáveis cardíacas, o máximo valor obtido de frequência cardíaca em repouso se correlacionou com a média dos intervalos RR da VFC ( $r=-0,70 ; p<0,001)$. Além deste, com exceção da ausência de correlação entre PNN50 e VLF $(r=0,13 ; p=0,5)$ e entre LF e LF/HF $(r=$ 0,01; $\mathrm{p}=0,9)$, as variáveis SDNN, RMSSD, PNN50, VLF, LF, HF, LF/HF, SD1 e SD2 se correlacionaram de modo significante, com $r$ entre 0,42 e 0,99 (respectivamente $p=0,02$ e $p<$ 0,001). As relações entre diferentes variáveis são apresentadas na tabela 3 . Na figura 1 são apontadas as correlações entre número máximo de repetições no teste de resistência abdominal e diferentes variáveis da VFC, relacionadas ao domínio do tempo (painéis A e B) e não-linear (painéis C e D). 
Tabela 3. Dados de correlação entre variáveis eleitas no estudo com militares no Núcleo de Preparação Física de Oficiais da Reserva de Pelotas/RS (n=30).

\begin{tabular}{llrc}
\hline & & $\mathrm{r}$ & $\mathrm{p}$ \\
\hline Massa Corporal & \%GC & 0,78 & $<0,001$ \\
& FIPMD & 0,39 & 0,02 \\
& FIPME & 0,57 & 0,001 \\
& LF/HF & 0,47 & 0,009 \\
\multirow{5}{*}{ Salto Vertical } & FIPMD & 0,40 & 0,02 \\
& FIPME & 0,53 & 0,002 \\
& Salto Vertical & $-0,47$ & 0,009 \\
Salto horizontal & Salto horizontal & 0,45 & 0,01 \\
& Corrida de 20 m & $-0,37$ & 0,04 \\
& Flexão de braço & 0,41 & 0,02 \\
& Corrida de 20 m & $-0,36$ & 0,04 \\
& Flexão de braço & 0,40 & 0,02 \\
FC de repouso - média & Barra fixa & 0,47 & 0,008 \\
& PNN50 & 0,46 & 0,009 \\
& LF/HF & $-0,44$ & 0,01 \\
& FC de repouso - máxima & 0,67 & $<0,001$ \\
& MedRR & $-0,98$ & $<0,001$ \\
& RMSSD & 0,45 & 0,01 \\
& PNN50 & $-0,67$ & $<0,001$ \\
MedRR & SD1 & $-0,45$ & 0,01 \\
& RMSSD & 0,42 & 0,02 \\
& PNN50 & 0,64 & $<0,001$ \\
& SD1 & 0,42 & 0,02 \\
\hline
\end{tabular}

$\% \mathrm{GC}=$ gordura corporal; FIPMD = Força isométrica de preensão manual direita; FIPME = Força isométrica de preensão manual esquerda; PNN50 = Percentual dos intervalos entre batimentos com mais de $50 \mathrm{~ms}$ em relação ao subsequente; LF/HF = Razão entre LF e HF; MedRR = média dos intervalos entre batimentos; RMSSD = Raiz média do desvio padrão dos batimentos cardíacos; SD1 $=$ Desvio padrão entre pontos instantâneos. 

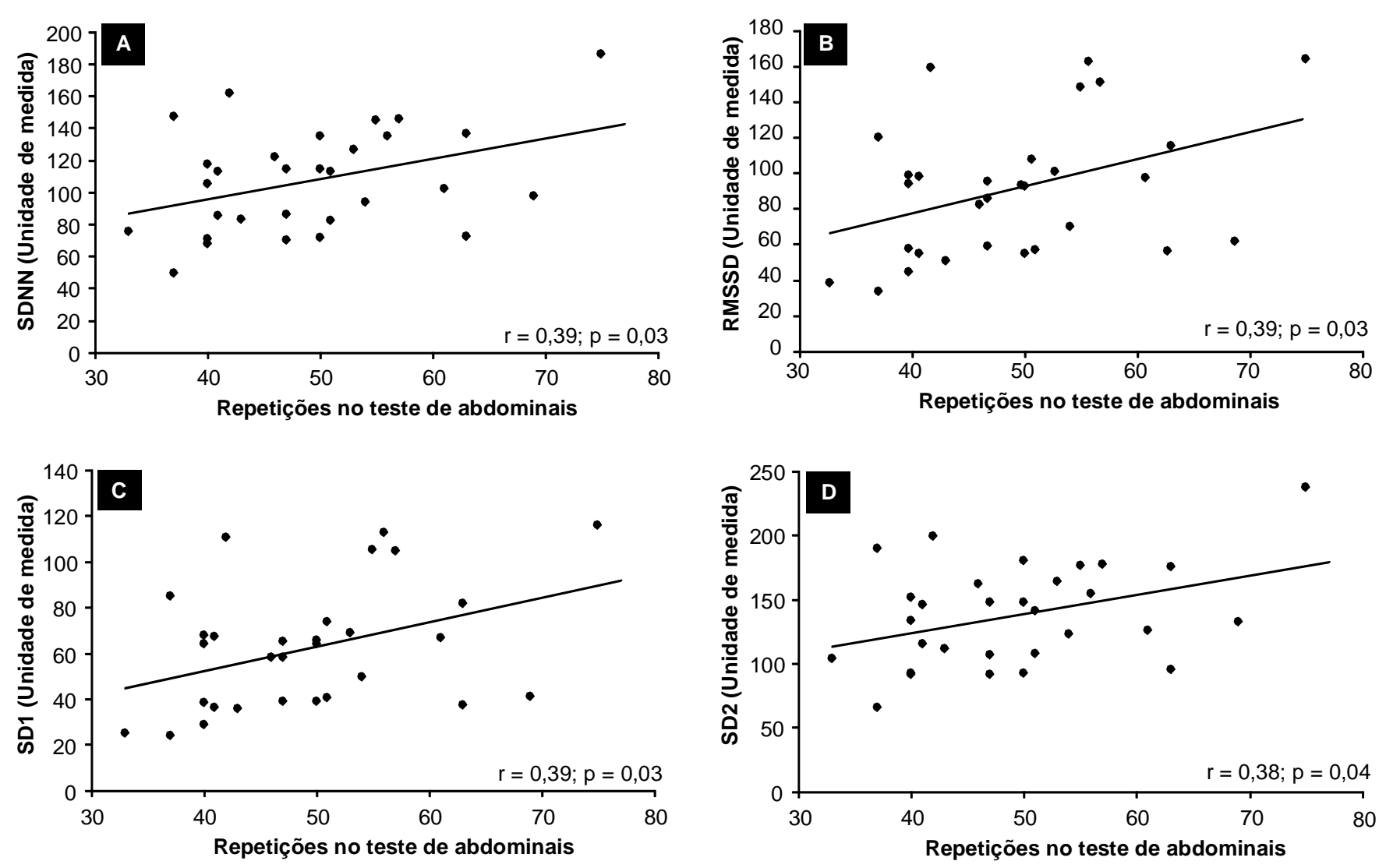

Figura 1. Correlações entre desempenho no teste de abdominais e medidas da VFC de militares no Núcleo de Preparação Física de Oficiais da Reserva de Pelotas/RS (n=30).

\section{Discussão}

Este estudo buscou avaliar e testar as relações entre aptidão física, concentração de CK e níveis de variabilidade da frequência cardíaca em jovens militares ingressantes do NPOR. Destacam-se, como achados relevantes: i) diferentes correlações entre VFC e desempenho nos testes de salto horizontal e de abdominais; ii) ausência de relação entre [CK] e VO2max com as demais variáveis.

Registra-se que, quanto a composição corporal, os envolvidos exibiam baixa quantidade de gordura (DEURENBERG; WESTSTRATE; SEIDELL et al., 1991), inferior à observada em outros militares brasileiros (VARGAS; MOLETA; PILATTI et al., 2013). Acerca da aptidão cardiorrespiratória, destaca-se que os sujeitos do presente estudo exibiram $\mathrm{VO}_{2 \text { máx }}$ superior aos militares de outro estado nacional (CERRIANI et al., 2012), mas ligeiramente inferior ao de croatas da terceira geração de militares recrutas mais velhos, com 29,10 \pm 5,6 anos (SPORIS, 2013). Indicase que o desenvolvimento deste componente da aptidão física é relevante para reduzir riscos de 
doenças cardíacas e obesidade, e eleva a capacidade de realizar tarefas que envolvam longa duração e resistência (KYROLAINEN et al., 2008).

Na perspectiva neuromuscular, o desempenho no teste de flexibilidade se encontra dentro da média (ACSM, 2007). Quanto ao salto horizontal, medida síntese da potência de membros inferiores, os valores do presente estudo se apresentam superiores $(185,1 \pm 15,16 \mathrm{~cm})$, aos $152 \pm 43$ $\mathrm{cm}$ observados entre jovens (CASTRO-PIÑERO; ORTEGA; ARTERO, 2010) e inferiores aos mais de $220 \mathrm{~cm}$ encontrados em praticantes de Brazilian Jiu-Jitsu (COSWIG; NEVES; DEL VECCHIO; 2011). Embora isto não seja muito problemático, valores superiores e intervenções que constituam de alongamento, fortalecimento e pliometria podem reduzir a incidência de lesões entre militares (BULLOCK et al., 2010). Foi registrada FIPM da mão direita e esquerda de 42,84 $\pm 8,05 \mathrm{kgf}$ e $41,15 \pm 7,56 \mathrm{kgf}$, respectivamente, mostrando-se menor que a média de mão dominante (44,2 kgf) e maior que a média de mão não dominante $(40,05 \mathrm{kgf})$ observadas no estudo de Caporrino et al. (1998). Atletas de taekwondo revelaram valores médios de mão dominante superiores, de

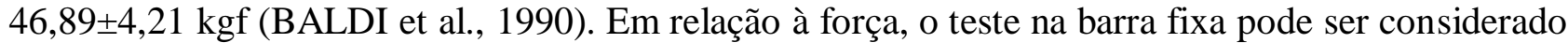
instrumento confiável de medida para testar a força e a resistência de membros superiores (MARTINS, 2004). Estudo de Ceriani et al. (2008), também com alunos do NPOR, mas de Bayeux/PB, explicitou média de 12 repetições máximas de barra fixa e flexibilidade de $32 \pm 8,1 \mathrm{~cm}$, indicando força média dos sujeitos do presente estudo superior aos alunos do NPOR de Bayeux/PB; porém, com nível de flexibilidade inferior.

Medidas de CK são relevantes para avaliar lesão muscular (HELED et al., 2007) e com o conhecimento dos valores de $\mathrm{CK}$, consegue-se analisar a intensidade da lesão no músculo esquelético e do treinamento físico (TOTSUKA et al., 2002). Assume-se como valor limite $500 \mathrm{U} \cdot \mathrm{L}^{-}$ ${ }^{1}$ para atletas não lesionados (PETIBOIS, 2002), e a maioria dos militares do presente estudo está dentro deste limite. Porém, alguns indivíduos (20\%) exibiram [CK] superior (521 U/L a $1900 \mathrm{U} / \mathrm{L}$ ). Em contrapartida às $500 \mathrm{UL}^{-1}$, atletas mostram CK de repouso alta, se comparados a pessoas sedentárias, mas o aumento após o exercício é menor (BRANCACCIO; MAFFULLI; LIMONGELLI, 2007). Em jogadores profissionais de futebol, por exemplo, a [CK] é de 192,1 \pm 23,0 U.L $\mathrm{L}^{-1}$ no momento pré-jogo, mas chega a $785 \pm 95,5 \mathrm{U} \cdot \mathrm{L}^{-1}$ no pós-jogo (COELHO et al., 2011). Na perspectiva militar, estudo envolvendo 499 recrutas dos EUA mostrou que após treinamento intenso, soldados afro-americanos apresentavam valores de CK de $1352 \pm 1102,8 \mathrm{U} \cdot \mathrm{L}^{-}$ ${ }^{1}$, concentrações elevadas para os valores de referência (LANDAU et al., 2012).

Quanto aos índices relacionados à VFC, sejam estes no domínio do tempo ou no domínio da frequência, os altos valores apresentados indicam haver funcionamento adequado dos mecanismos autonômicos. Sendo a VFC marcador do balanço autonômico, a correlação entre a VFC e os valores da FC está baseada no fato da FC aumentar em função da maior ativação do ramo simpático e reduzir seus valores frente ao aumento da atividade parassimpática (AUBERT; SEPS; BECKER, 2003; BARTH; DEL VECCHIO, 2014). A partir da VFC e da variação de desempenho em testes motores, Oliveira et al. (2012) identificaram que atletas de futebol que apresentaram maior ativação vagal foram mais responsivos ao treinamento, porém, os autores indicam que os mecanismos de aumento do desempenho relacionado ao aumento da ativação vagal ainda são desconhecidos.

Entre adultos jovens brasileiros foi observada correlação significante $(r=-0,65)$ entre domínio da frequência da VFC (delta de variação da banda HF da posição ortostática para supina) e potência aeróbia máxima estimada por questionário, também já se observaram relações estatisticamente significantes entre VFC e medidas diretas de aptidão aeróbia máxima 
(BUCHHEIT; GINDRE, 2006), embora parâmetros da VFC expliquem pouco a variância na aptidão aeróbia máxima (GRANT et al., 2009). Tais relações se dão pois sujeitos que exibem maior aptidão aeróbia tendem a apresentar melhor ajustamento da modulação cardíaca adrenérgica e vagal (GONÇALVES et al., 2014). No entanto, assim como já observado previamente, destaca-se que esta relação não foi observada no presente estudo, provavelmente porque perfil de VFC favorável, como elevados níveis de HF e valores superiores nas demais variáveis da VFC se constituem mais como condição favorável para o aumento da potência aeróbia máxima do que valores elevados da mesma (MARTINELLI et al., 2005). Ademais, outros fatores podem explicar esta ausência de correlação, como ajuste mais rápido do consumo máximo de oxigênio em relação às modificações dos parâmetros de VFC (LEICHT et al., 2003; BOSQUET, GAMELIN, BERTHOIN 2007), em especial a partir de modificações periféricas, como vascularização, capilarização e funcionamento enzimático (PERINI et al., 2002; HEDELIN; BJERLE; HENRIKSSON-LARSEN, 2001).

Assim como no presente estudo, que constatou relação entre HF/LH e potência de membros inferiores, bem como correlação entre SD1, SD2, SDNN e RMSSD com desempenho no teste de abdominais, investigação prévia também registrou relação entre VFC e força muscular, com $\mathrm{r}=$ 0,82; $\mathrm{p}=0,04$ (BRETZ et al., 2014), embora mais estudos sejam necessários para o melhor entendimento desta relação.

De acordo com Martins (2004), militares devem exibir aptidão física excelente, sendo capazes de sustentar e deslocar seu próprio corpo, o que aumenta a chance de sucesso de suas missões. Assim, o presente estudo mostrou as condições físicas dos militares de um curso de formação a oficial temporário brasileiro. Neste sentido, até o período de coleta, o treinamento pareceu não ser suficiente para elevar os níveis de aptidão física. Em estudos futuros, sugere-se avaliação dos parâmetros estudados na presente investigação em momentos pré e pós exercícios de longa duração, também conhecidos como exercícios de campo, para verificar o efeito deste tipo de exercício na aptidão física, respostas fisiológicas e aspectos psicológicos de militares.

\title{
Conclusões
}

Conclui-se, através dos dados da presente investigação, que o nível de aptidão física dos militares envolvidos é intermediário. A concentração de CK está próxima ao limite superior de referência e os valores da variabilidade da frequência cardíaca indicam funcionamento adequado dos mecanismos autonômicos.

Ademais, foram observadas correlações significantes entre testes neuromusculares e variabilidade da frequência cardíaca. As correlações esperadas entre CK e potência aeróbia e entre estas com a variabilidade da frequência cardíaca não foram identificadas.

\section{RELATIONSHIPS BETWEEN PHYSICAL FITNESS, CREATINE KINASE CONCENTRATION AND HEART RATE VARIABILITY IN STUDENTS FROM NPOR OF PELOTAS/RS.}

\begin{abstract}
:
The objective of this study was to evaluate the profile of active military officials from the Reserve Officers Training Center (NPOR) of Pelotas-RS. The study engaged 30 militaries who did daily
\end{abstract}


physical training. Researchers measured lower limb power, speed, strength, aerobic power $\left(\mathrm{VO}_{2 \max }\right)$, heart rate variability (HRV), and creatine kinase blood concentration ([CK]). For correlations, researchers used Pearson's coefficient. Those involved showed $66.9 \pm 7.2 \mathrm{~kg}$, rest [CK] was $390.7 \pm 317.9 \mathrm{U} \cdot \mathrm{L}^{-1}$ and $\mathrm{VO}_{2 \max }$ of $48.85 \pm 5.99 \mathrm{~mL} \cdot \mathrm{kg}^{-1} \cdot \mathrm{min}^{-1}$. Researchers concluded that the physical fitness of participating military is intermediate. The CK blood concentration is near the upper limit of reference, and values of HRV indicate proper autonomic functioning.

Keywords: Physical fitness. Physical education and training. Military training. Creatine kinase. Heart rate.

\section{RELACIONES ENTRE LA CONDICIÓN FÍSICA, LA CONCENTRACIÓN DE CREATINA QUINASA Y LA VARIABILIDAD DE LA FRECUENCIA CARDÍACA EN LOS ESTUDIANTES NPOR DE PELOTAS/RS.}

\section{Resumen:}

El objetivo fue evaluar el perfil de militares activos del Centro de Preparación de Oficiales de Pelotas/RS. Estuvieron incluidos 30 militares que participan del entrenamiento físico diario. se midieron el poder de los miembros inferiores, velocidad, resistencia de fuerza, la capacidad aeróbica $\left(\mathrm{VO}_{2 \max }\right)$, variabilidad de la frecuencia cardiaca (VFC) y la concentración de creatina quinasa ([CK]). Los estadísticos descriptivos y las correlaciones fueron probados con la prueba de Pearson. Los sujetos tenían $66.9 \pm 7.2 \mathrm{~kg}$, la [CK] fue $390,7 \pm 317,9 \mathrm{U} \cdot \mathrm{L}^{-1}$ y VO2max de 48,85 $\pm 5,99$ $\mathrm{ml} \bullet \mathrm{kg}^{-1} \cdot \mathrm{min}^{1}$. Se concluye que la condición física del personal militar involucrado es intermedio. La concentración de CK está cerca del límite superior de referencia y los valores de la VFC indican funcionamiento autonómico adecuado.

Palabras-clave: Aptitud física. Educación física y entrenamiento. Entrenamiento militar. Creatinaquinasa. Frecuencia cardiaca.

\section{Referências}

AUBERT, A.E.; SEPS, B.; BECKERS, F. Heart rate variability in athletes. Sports Medicine, Auckland, NZ, v.12, n.33, p.889-919, 2003.

BALDI, M.; DIANNO, M.V.; ANDRADE, D.R.; PEREIRA, M.H.N. Comparação de aptidão física em atletas de Tae-kwon-do masculino em 2 diferentes níveis. Revista Brasileira de Ciências do Movimento, v.3, n.4, p.26-33, jul, 1990.

BARTH, J.; DEL VECCHIO, F.B. Efeitos da frequência ventilatória sobre os índices da variabilidade da frequência cardíaca. Revista Iberoamericana de Arritmología, v.5, n.1, p.185193, 2014.

BARTONE, P.T.; ROLAND, R.R.; PICANO, J.J.; WILLIAMS, T.J. Psycological hardiness predicts success in US Army Special Forces Candidates. International Journal of Selection and Assessment, n.1, v.16, p.78-81, mar, 2008. 
BOSQUET, L.; GAMELIN, F.X.; BERTHOIN, S. Is aerobic endurance a determinant of cardiac autonomic regulation? European Journal of Applied Physiology, v.100, p.363-369, 2007.

BRANCACCIO, P.; MAFFULLI, N.; LIMONGELLI, F.M. Creatine kinase monitoring in sport medicine. British Medical Bulletin, v.81-82, n.14, p. 209-230, jun, 2007.

BRETZ, E.; KÓBOR-NYAKAS, D.E.; BRETZ, K.J.; HREHUSS, N.; RADÁK, Z.; NYAKAS, C. Correlations of psycho-physiological parameters influencing the physical fitness of aged women. Acta Physiologica Hungarica, v.8, p. 1-8, set, 2014.

BUCHHEIT, M.; GINDRE, C. Cardiac parasympathetic regulation: respective associations with cardiorespiratory fitness and training load. Heart Circulation Physiology, v.1, n.291, p. 451-458, jul, 2006.

BULLOCK, S.H.; JONES, B.H.; GILCHRIST, J.; MARSHALL, S.W. Prevention of physical training-related injuries recommendations for the military and other active Populations based on expedited systematic reviews. American Journal of Preventive Medicine, v.1, n.38, p.156-181, jan, 2010.

CAPORRINO, F.A.; FALOPPA, F; SANTOS, J.B.G. et al. Estudo populacional da força de preensão palmar com dinamômetro Jamar. Revista Brasileira de Ortopedia, n.2, v.33, 1998.

CARTER, J.B.; BANISTER, E.W.; BLADER, A.P. Effect of endurance exercise on autonomic control of heart rate. Sports Medicine, Auckland, v.33, n.1, p.33-46, 2003.

CASTRO-PIÑERO, J.; ORTEGA, F.B., ARTERO, E.G.; GIRELA-REJÓN, M.J.; MORA, J.; SJÖSTRÖM, M.; RUIZ, J.R. Assessing muscular strength in youth: usefulness of standing long jump as a general index of muscular fitness. Journal of Strength and Conditioning Research, v.7, n.24, p.1810-1817, jul, 2010.

CERIANI, R.B.; PONTES, L.M.; CARDOSO, AB.; GOMES, ALM.; DANTAS, E.H.M. Impacto do treinamento físico militar sobre os níveis de aptidão física de alunos do núcleo preparatório de oficiais da reserva (NPOR). Revista da AMRIGS, Porto Alegre, v.3, n.52, p. 164-169, jul, 2008.

COELHO, D.B.; MORANDI, R.F.; MELO, M.A.A.; GARCIA, E.S. Cinética da creatina quinase em jogadores de futebol profissional em uma temporada competitiva. Revista Brasileira de Cineantropometria e Desempenho Humano, v.3, n.13, p.189-194, 2011.

COSWIG, V.S.; NEVES, A.H.S.; DEL VECCHIO, F.B. Características físicas e desempenho motor no jiu-jitsu brasileiro: estudo com iniciantes e experientes na modalidade. Lecturas, Educación Física y Deportes-Revista digital, Buenos Aires, v.16, n.162, nov, 2011. Disponível em <http://www.efdeportes.com/efd162/desempenho-motor-no-jiu-jitsu-brasileiro.htm>. Acesso em: out 2014. 
DEURENBERG, P.; WESTSTRATE, J.A.; SEIDELL, J. Body mass index as a measure of body fatness, age- and sex-specific prediction formulas. British Journal of Nutrition, v.65, n.2, p.105114, 1991.

DREGVAL, L.; VAIČAITIENĖ, R. Anthropometrical data and physical fitness of Lithuanian soldiers according to the sociodemographic characteristics. Medicina, Kaunas, v.1, n.42, p.57-63, 2006.

DYRSTAD, SM.; SOLTVEDT, R.; HALLE`N, J. Physical Fitness and Physical Training during Norwegian Military Service. Military Medicine, v.8, n.171, p.736-741, 2006.

ERLACHER, E. Os impactos das ações da polícia militar do espírito santo em regiões urbanas violentas: o caso flexal. Revista Preleção, v.5, n.5, p.105-131, abr, 2009.

GONÇALVES, T.R.; FARINATTI, P.; GURGEL, J.L.; SOARES, P.P.S. Correlation between cardiac autonomic modulation in response to orthostatic stress and indicators of quality of life, physical capacity, and physical activity in healthy individuals. Journal of Strength and Conditioning Research, n.26, nov, 2014.

GRANT, C.C.; CLARK, J.R.; RENSBURG, D.C.; VILJOEN, M. Relationship between exercise capacity and heart rate variability: supine and in response to an orthostatic stressor. Autonomic Neuroscience, v.2, n.151, p.186-188, dez, 2009.

HARMAN, E.; GUTEKUNST, D.J.; FRYKMAN, P.N. Effects of two different eight-week training programs on military physical performance. Journal of Strength and Conditioning Research, v.2, n.22, p.524-524, 2008.

HARTMANN, E.; SUNDE, T.; KRISTENSEN, W.; MARTINUSSEN, M. Psychological measures as predictors of military training performance. Journal of Personality Assessments, v.1, n.80, p.87-98, 2003.

HEDELIN, R.; BJERLE, P.; HENRIKSSON-LARSEN, K. Heart rate variability in athletes: relationship with central and peripheral performance. Medicine and Science Sports Exercise, v.33, n.8, p.1394-1398, 2001.

HELED, Y.; BLOOM, M.S.; WU, T.J.; STEPHENS, Q.; DEUSTER, PA. CM-MM and ACE genotypes and physiological prediction of the creatine kinase response to exercise. Journal of applied Physiology, v.103, p.503-510, 2007.

HENDERSON, N.; KNAPIK, J.; SHAFFER, S.; MCKENZIE, T.; SCHNEIDER, G. Injuries and injury risk factors among men and women in US Army combat medic advanced individual training. Military Medicine, v.9, n.165, p.647-652, 2000. 
JACKSON, A.S.; POLLOCK, M.L. Generalized equations for predicting body density of men. British Journal of Nutrition, Houston, v.40, p.497-504, 1978.

JUNIOR, W.E.P; Krug, M.R. Aptidão física e qualidade de vida dos oficiais e sargentos do $29^{\circ}$ grupo de artilharia de campanha auto-propulsado de Cruz Alta, RS. Lecturas, Educación Física y Deportes- Revista Digital, Buenos Aires, v.16, n.155, 2011. Disponível em: <http://www.efdeportes.com/efd155/aptidao-fisica-dos-oficiais-e-sargentos.htm>. Acesso em: out, 2014.

KYROLAINEN, H.; HAKKINEN, K.; KAUTIAINEM, H.; SANTILLA, M.; PIHLAINEN, K.; HAKKINEN, A. Phyical fitness, BMI and Sickness absence in male military personnel. Occupacional Medicine, n.58, p.251-256, 2008.

LANDAU, M.E.; KENNEY, K.; DEUSTER, P.; GONZALEZ, R.S.; CONTRERAS-SESVOLD, C.; SAMBUUGHIN, N.; O'CONNOR, F.G.; CAMPBELL WW.. Investigation of the relationship between serum creatine kinase and genetic polymorphisms in military recruits. Military Medicine, v.11, n.177, p.1359-1365, nov, 2012.

LEICHT, A.S.; ALLEN, G.D.; HOEY, A.J. Influence of intensive cycling training on heart rate variability during rest and exercise. Canadian Journal of Applied Physiology, v.28, n.6, p.898909. 2003.

LEVANDOSKI, G.; CHIQUITO, E.; OLIVEIRA, A.G.; ZEREMBA, C.M. Qualidade de vida e composição corporal de soldados ingressantes no exército. Revista Brasileira de Qualidade de Vida, v.2, n.5, p.23-30, 2013.

LINDQUIST, C.H.; BRAY, R.M. Trends in overweight and physical activity among U.S. military personnel, 1995-1998. Preventive Medicine, v.1, n.32, p.57-65, 2001.

MARKOVIC, G.; DIZDAR, D.; JUKIC, I.; CARDINALE, M. Reliability and factorial validity of squat and countermovement jump tests. Journal of Strength and Conditional Research, v.18, n.3, p.551-555, 2004.

MARTINELLI, F.S.; CHACON-MIKAHIL, M.P.T.; MARTINS, L.E.B.; LIMA-FILHO, E.C.; GOLFETTI, R.; PASCHOAL, M.A.; GALLO-JUNIOR, L. Heart rate variability in athletes and non-athletes at rest and during head-up tilt. Brazilian Journal of Medicine and Biology Research [online], v.38, n.4, p.639-647, 2005.

MARTINS, M.E.A.; DOS SANTOS, F.M.; ARANTES, R.P.; ALVES, C.S.; MIGUEL, L.B.; BASTOS, J.F.A.; DA SILVA, S.A.A.; GUILARDUCCI, R.V.; LA PORTA JR, M.A.M.; DA SILVA, E.B. Relação da performance na barra fixa com a força de preensão manual e tempo de sustentação na barra fixa. Revista de Educação Física, v.128, p.65-71, Rio de Janeiro, 2004. 
MEDICAL SURVEILLANCE MONTHLY REPORT- MSMR. Update: exertional rhabdomyolysis, active component, U.S. Armed Forces, 2012.

OLIVEIRA, R.S.; PEDRO, R.E.; MILANEZ, V.F.; BORTOLOTTI, H.; COSTA, M.V.; NAKAMURA, F.Y. Relação entre variabilidade da frequência cardíaca e aumento no desempenho físico em jogadores de futebol. Revista Brasileira de Cineantropometria do Desempenho Humano, n.6, v.14, p.713-722, 2012.

PERINI, R.; FISHER, N.; VEICSTEINAS, A.; PENDERGAST, D.R. Aerobic training and cardiovascular responses at rest and during exercise in older men and women. Medicine and Science in Sports Exercise, v.34, n.4, p.700-708, 2002.

PETIBOIS, C.; CAZORLA, G.; POORTMANS, JR.; DÉLERIS, G. Biochemical aspects of overtraining in endurance sports. Sports Medicine, v.13, n.32, p.867-878, 2002.

PIMENTA, E.M. Polimorfismos genéticos para alfa actinina 3 e sua relação com dano muscular e capacidades funcionais de atletas de futebol. 2012, 193p. Tese (Mestrado), Universidad de León, 2012.

PUMPRLA, J.; HOWORKA, K.; GROVES, D.; CHESTER, M.; NOLAN, J. Functional assessment of heart rate variability: physiological basis and practical applications. International Journal of Cardiology, v.1, n.84, p.1-14, 2002.

RESENDE, L.; GONÇALVES, P. As operações de paz das Nações Unidas e o Brasil. A apresentação de um paradoxo político. In: II SIMPÓSIO DE PÓS-GRADUAÇÃO EM RELAÇÕES INTERNACIONAIS DO PROGRAMA "SAN TIAGO DANTAS", São Paulo-SP, 2009, v.1, p.1-16.

ROBERT, L.; NEWTON, J.R.; HONGMEI, H.; STEWART, T.M.; RYAN, D.H.; WILLIAMSON, D.A. Efficacy of a pilot internet-based weight management program (h.e.a.l.t.h.) and longitudinal physical fitness data in army reserve soldiers. Journal Diabetes Science Technology, v.5, n.5, p.1255-1262, 2011.

SEITENFUS, R. De Suez ao Haiti: a participação brasileira nas missões de paz. webdoc: seitenfus.com.br, $2006 \mathrm{sept}$; 1-15.

SIRI, W.E. Body composition from fluid spaces and density: analysis of methods. In: Brozeck J, Henschel A eds. Techniques for measuring body composition. Washington DC: National Academy of Sciences. Natural Resource Council, p. 223-244, 1961.

SPORIS, G. Validity of 2-mile run test for determination of $\mathrm{VO}_{2}$ max among soldiers. Journal of Sport and Human Performance, v.1, n.1, p.15-22, 2013. 
THOMAS, J.L.; ADLER, A.B.; WITTELS, P.; ENNE, R.; JOSHANNES, B. Comparing elite soldier's perceptions of psychological and physical demands during military training. Military Medicine, v.7, n.169, p.526-530, jul, 2004.

TOTSUKA, M.; NAKAJI, S.; SUZUKI, K.; SUGAWARA, K.; SATO, K. Break point of serum creatine kinase release after endurance exercise. Journal of Applied Physiology, v.4, n.93, p. 2801286, out, 2002.

VANDERLEI, L.C.M.; PASTRE, C.M.; HOSHI, R.A.; CARVALHO, T.D.; GODOY, M.F. Noções básicas de variabilidade da frequência cardíaca e sua aplicabilidade clínica. Revista Brasileira de Cirurgia Cardiovascular, v.2, n.24, p.205-217, 2009.

VARGAS, L.M.; MOLETA, T.; PILATTI, L.A. Diferença da aptidão física relacionada ao desempenho entre soldados de elite e convencionais do exército brasileiro. Revista da faculdade de Educação Física da UNICAMP, v.2, n.11, p.148-167, jun, 2013.

WELLS, K.F.; DILLON, E.K. The sit and reach: a test of back and leg flexibility. Research Quarterly for Exercise and Sport, Washington, v.23, p.115-118, 1952.

WELTMAN, J.; SEIP, R.; LEVINE, S.; SNEAD, D.; KAISER, D.; ROGOL, A. Prediction of lactate threshold and fixed blood lactate concentrations from 3200-m time trial running performance in untrained females. International Journal of Sports Medicine, v.10, p.207-211, 1989.

ZANCHET, M.A.; DEL VECCHIO, F.B. Efeito da Kinesio Taping sobre força máxima e resistência de força em padelistas. Fisioterapia do movimento, Curitiba, v.26, n.1, Mar, 2013.

Recebido em: 25/02/2015

Revisado em: 04/05/2015

Aprovado em: 07/05/2015

Endereço para correspondência:

Fabrício Boscolo Del Vecchio

fabricio_boscolo@uol.com.br

Universidade Federal de Pelotas, Escola Superior de Educação Física.

Rua Luiz de Camões, 625

Cohab Tablada

96055-630 - Pelotas, RS - Brasil 